\title{
Exchange interaction and its tuning in magnetic binary chalcogenides
}

\author{
M. G. Vergniory, ${ }^{1,2,3,{ }^{*}}$ M. M. Otrokov, ${ }^{1,2,3}$ D. Thonig, ${ }^{1}$ M. Hoffmann, ${ }^{1,4}$ I. V. Maznichenko, ${ }^{4}$ M. Geilhufe, ${ }^{1}$ X. Zubizarreta,,${ }^{1,2}$ \\ S. Ostanin, ${ }^{1}$ A. Marmodoro, ${ }^{1}$ J. Henk, ${ }^{4}$ W. Hergert, ${ }^{4}$ I. Mertig, ${ }^{1,4}$ E. V. Chulkov, ${ }^{2,3,5}$ and A. Ernst ${ }^{1,6, \dagger}$ \\ ${ }^{1}$ Max-Planck-Institut für Mikrostrukturphysik, Weinberg 2, D-06120 Halle, Germany \\ ${ }^{2}$ Donostia International Physics Center, P. Manuel de Lardizabal 4, San Sebastián, 20018 Basque Country, Spain \\ ${ }^{3}$ Tomsk State University, pr. Lenina 36, 634050 Tomsk, Russia \\ ${ }^{4}$ Institut für Physik, Martin-Luther-Universität Halle-Wittenberg, D-06099 Halle, Germany \\ ${ }^{5}$ Departamento de Física de Materiales, Facultad de Ciencias Químicas, Apdo. 1072, San Sebastián, 20080 Basque Country, Spain \\ ${ }^{6}$ Wilhelm-Ostwald-Institut für Physikalische und Theoretische Chemie, Universität Leipzig, Linnéstraße 2, D-04103 Leipzig, Germany
}

(Received 26 June 2013; revised manuscript received 3 February 2014; published 16 April 2014)

\begin{abstract}
Using a first-principles Green's function approach we study magnetic properties of the magnetic binary tetradymite chalcogenides $\mathrm{Bi}_{2} \mathrm{Se}_{3}, \mathrm{Bi}_{2} \mathrm{Te}_{3}$, and $\mathrm{Sb}_{2} \mathrm{Te}_{3}$. The magnetic coupling between transition-metal impurities is long range, extends beyond a quintuple layer, and decreases with increasing number of $d$ electrons per $3 d$ atom. We find two main mechanisms for the magnetic interaction in these materials: the indirect exchange interaction mediated by free carriers and the indirect interaction between magnetic moments via chalcogen atoms. The calculated Curie temperatures of these systems are in good agreement with available experimental data. Our results provide deep insight into exchange interactions in magnetic binary tetradymite chalcogenides and open a way to design new materials for promising applications.
\end{abstract}

DOI: 10.1103/PhysRevB.89.165202

PACS number(s): 72.15.Jf, 71.70.Ej, 85.80.Fi

\section{INTRODUCTION}

Binary tetradymite chalcogenides, in particular $\mathrm{Bi}_{2} \mathrm{Se}_{3}$, $\mathrm{Bi}_{2} \mathrm{Te}_{3}$, and $\mathrm{Sb}_{2} \mathrm{Te}_{3}$, are of great interest due to their outstanding structural and electronic properties. These compounds consist of repeated blocks of five atomic layers (quintuple layers, QLs) separated by the van der Waals gaps. Their electronic structure features a narrow band gap and strong spinorbit coupling, which are responsible for the inverted band structure at the Brillouin zone center. Because of the high figure of merit at room temperature tetradymite chalcogenides are attractive for thermoelectric applications [1]. At the end of the last decade, topologically protected states have been observed at the surfaces of all aforementioned compounds, which makes them a subject of intense research [2,3]. Being already well studied, nowadays binary tetradymite chalcogenides serve as a basis for new materials or hybrid structures featuring topological properties [4-12]. This is feasible by stacking of building blocks of different compounds [4-6], making heterostructures $[7,8]$, or specific doping [9-12]. The case of magnetic doping is especially promising, since it allows us to achieve the quantum anomalous Hall effect [12], which is expected to pave a way to the low-power-consumption electronic devices.

The focus of the present work is on the magnetic properties of binary tetradymite chalcogenides doped with $3 d$ transitionmetal impurities. Some of these compounds have already been studied as possible candidates for spintronics applications [13-23]. Most of the experiments were done on single crystals with a maximal dopant concentration of $x=0.1$. Stable ferromagnetic order was observed in chalcogenides doped with vanadium and chromium $[13,15,17]$, while samples doped with manganese were found to be either ferromagnetic at

\footnotetext{
*mgarcia@mpi-halle.de

†aernst@mpi-halle.de
}

very low temperatures or antiferromagnetic and paramagnetic depending on experimental conditions and sample preparation [14,16,21-23]. High Curie temperatures were reported for films prepared with molecular-beam epitaxy: 177 and $190 \mathrm{~K}$ for $\mathrm{Sb}_{1.65} \mathrm{~V}_{0.35} \mathrm{Te}_{3}$ and $\mathrm{Sb}_{1.41} \mathrm{Cr}_{0.59} \mathrm{Te}_{3}$, respectively $[19,20]$. The great interest in breaking time-reversal symmetry in topological insulators motivated further investigations of magnetic impurities located in particular at the surfaces of tetradymite chalcogenides [23-30].

Magnetic properties of transition-metal doped tetradymite chalcogenides can be efficiently described by first-principles methods. One of the first comprehensive studies was carried out by Larson and Lambrecht [31], who investigated the electronic and magnetic properties of bulk $\mathrm{Bi}_{2} \mathrm{Se}_{3}, \mathrm{Bi}_{2} \mathrm{Te}_{3}$, and $\mathrm{Sb}_{2} \mathrm{Te}_{3}$ doped with $3 d$ transition-metal atoms; their results for magnetically doped $\mathrm{Bi}_{2} \mathrm{Se}_{3}$ were confirmed by $\mathrm{Yu}$ et al. [32] and Zhang et al. [33]. Recently, it was shown that the Dirac surface state of the topological insulator $\mathrm{Bi}_{2} \mathrm{Te}_{3}$ survives upon moderate Mn doping of the surface QL, but can lose its topologically nontrivial character depending on the magnetization direction [34,35]. However, critical magnetic properties and the exchange interaction behavior in magnetic binary tetradymite chalcogenides were not studied in detail on a theoretical $a b$ initio level and, thus, are still under debate.

In this work, by using a first-principles Green's function method, we examine the electronic and magnetic structure as well as the exchange interaction mechanisms in the bulk tetradymite chalcogenides $\mathrm{Bi}_{2} \mathrm{Se}_{3}, \mathrm{Bi}_{2} \mathrm{Te}_{3}$, and $\mathrm{Sb}_{2} \mathrm{Te}_{3}$ doped by $3 d$ transition-metal atoms. We show that the exchange interaction in these materials can lead either to ferro- or antiferromagnetic order, depending on the host, concentration, and type of dopant. We identify the main mechanisms of the exchange interactions responsible for the onset of the long-range order, estimate critical temperature, and discuss ways to manipulate the magnetic properties of these systems. 


\section{COMPUTATIONAL DETAILS}

To study the magnetism of the $3 d$-metal-doped tetradymite chalcogenides we employed density functional theory within the generalized gradient approximation (GGA) [36]. According to test calculations, the results obtained by means of the local spin density approximation [37] differ marginally from those found with gradient-corrected functionals. We examined as well the local density approximation $+U(\mathrm{LDA}+U)$ approach for the treatment of correlation effects in these systems and obtained a good agreement with the previous study, which found only minor changes for $\mathrm{Co}$ and $\mathrm{Ni}$ impurities and valency stabilization of Fe impurities [31]. Because of the insignificant effect and to be consistent, we discuss here only results obtained with the GGA.

The electronic structure calculations were performed using a self-consistent full-charge-density Green's function method [38-40] in both fully relativistic and scalar-relativistic schemes for the experimental lattice parameters and atomic coordinates [41-43]. We used 24 Gaussian quadrature points to carry out a complex energy contour integration, while for the integration over the Brillouin zone we used a mesh of $20 \times 20 \times 10 \mathbf{k}$ points. Substitutional disorder was treated within the coherent potential approximation [44] (CPA).

The exchange constants $J_{i j}$ of the Heisenberg Hamiltonian

$$
\hat{H}=-\sum_{i, j} J_{i j} \vec{S}_{i} \cdot \vec{S}_{j}
$$

were obtained using the magnetic force theorem as it is implemented within multiple-scattering theory [45]. Here $i$ and $j$ are indices labeling magnetic atoms, while $\vec{S}_{i}$ and $\vec{S}_{j}$ are the magnetic moments of the $i$ th and $j$ th atoms, respectively. Inclusion of spin-orbit coupling leads to minor changes in the magnetic interaction (about 3\% to 5\% with respect to the scalar-relativistic case). Therefore, here we present only exchange constants calculated within the scalar-relativistic approximation. These exchange coupling parameters were then used as an input to Monte Carlo simulations which were utilized for the estimation of the critical temperatures.

According to the available experimental data [13-24,28,29], 3d-transition-metal impurities in bulk tetradymite chalcogenides substitute typically cation atoms (Bi and $\mathrm{Sb}$ ) and can supply 1 to 3 electrons for bonding. The comparably smaller size of transition-metal ions may furthermore lead to substantial relaxation of the underlying crystal structure [31]. We did not account for such structural deformations in our CPA calculations but investigated their impact on the magnetic interaction using a supercell approach and found only minor changes of the exchange constants. Therefore, the discussion below reports results from CPA calculations.

\section{RESULTS AND DISCUSSION}

\section{A. Exchange interaction}

We performed extensive calculations of $\mathrm{Bi}_{2-x} \mathrm{TM}_{x} \mathrm{Se}_{3}$, $\mathrm{Bi}_{2-x} \mathrm{TM}_{x} \mathrm{Te}_{3}$, and $\mathrm{Sb}_{2-x} \mathrm{TM}_{x} \mathrm{Te}_{3}(\mathrm{TM}=\mathrm{Ti}, \mathrm{V}, \mathrm{Cr}, \mathrm{Mn}, \mathrm{Fe}$, $\mathrm{Co}$, and $\mathrm{Ni}$ ) for the range of concentrations $0<x<1.0$. The electronic structure of these compounds calculated within the CPA agree for low and medium concentrations $(x<0.3)$

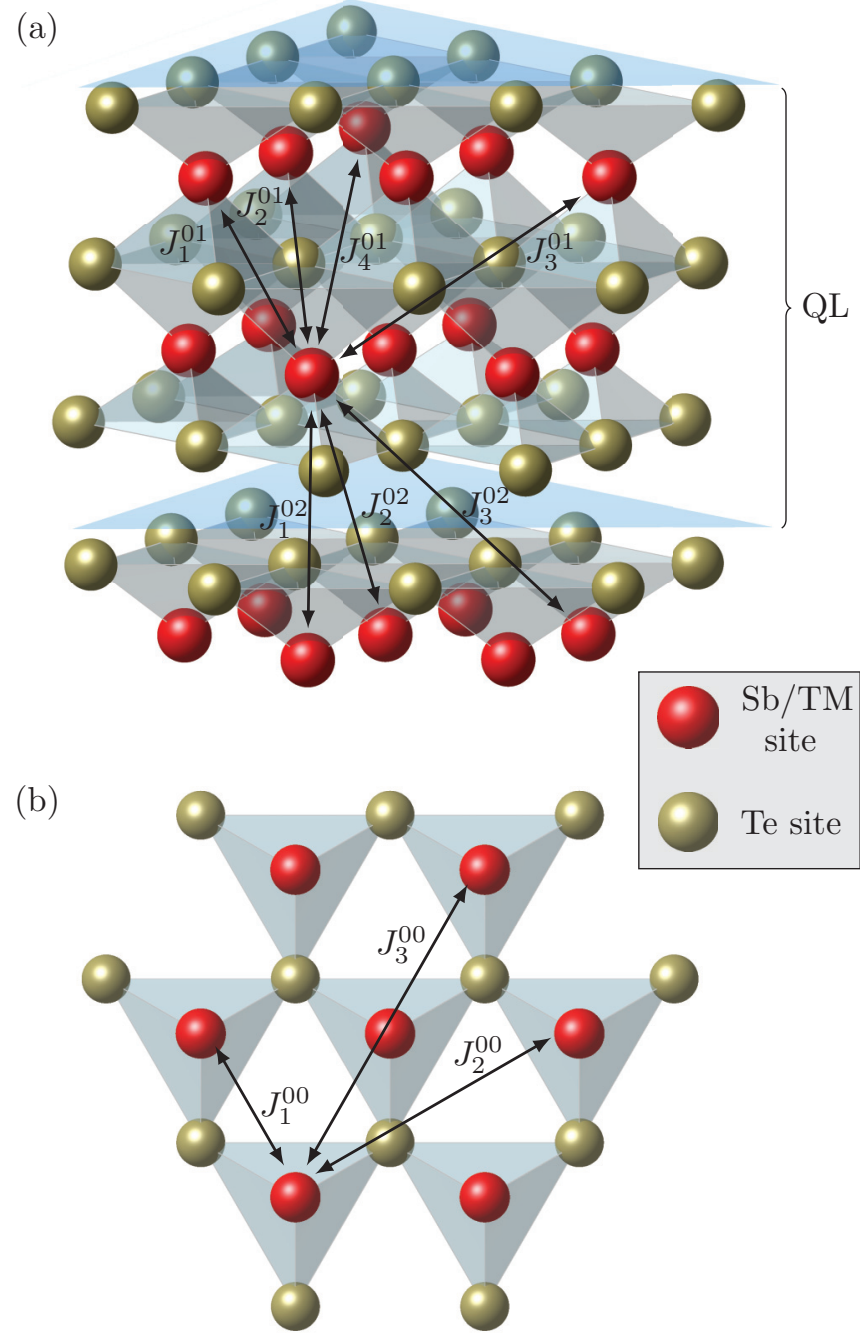

FIG. 1. (Color online) Schematic view of magnetic interactions in $\mathrm{Sb}_{2-x} \mathrm{TM}_{x} \mathrm{Te}_{3}$ : exchange interactions between (a) different layers and (b) within a single layer. The corresponding exchange constants for $\mathrm{Ti}, \mathrm{V}, \mathrm{Cr}, \mathrm{Mn}, \mathrm{Fe}$, and $\mathrm{Co}$ are shown in Fig. 2. The same plot applies to $\mathrm{Bi}_{2-x} \mathrm{TM}_{x} \mathrm{Se}_{3}$ and $\mathrm{Bi}_{2-x} \mathrm{TM}_{x} \mathrm{Te}_{3}$.

with those of previous supercell calculations by Larson and Lambrecht [31]. The self-consistently obtained Green's function was further used to calculate the exchange constants $J_{i j}$. Their relevant directions are depicted in Fig. 1 on top of the lattice structure of $\mathrm{Sb}_{2-x} \mathrm{TM}_{x} \mathrm{Te}_{3}$ for clarity, where we distinguish among in-plane (within the $\mathrm{Sb}$ or $\mathrm{Bi}$ plane) and out-of-plane coupling. Although experimental data are available for a large concentration range $[19,20]$, we first focus the present discussion on a representative value of $x=0.2$. The results presented in Fig. 2 can be summarized as follows:

(i) The effective exchange interaction is reduced with increasing number of $d$ electrons per TM atom, going from positive to negative values. The strongest ferromagnetic interaction is found between Ti atoms, which is explained by the density of states (DOS) of the impurities, as exemplified by $\mathrm{Sb}_{1.8} \mathrm{Ti}_{0.2} \mathrm{Te}_{3}$ [Fig. 3(a)]. The $d$ electrons of $\mathrm{Ti}$ atoms in the majority spin channel hybridize strongly with $s p$ states of the host. The corresponding DOS shows a lower localization, 

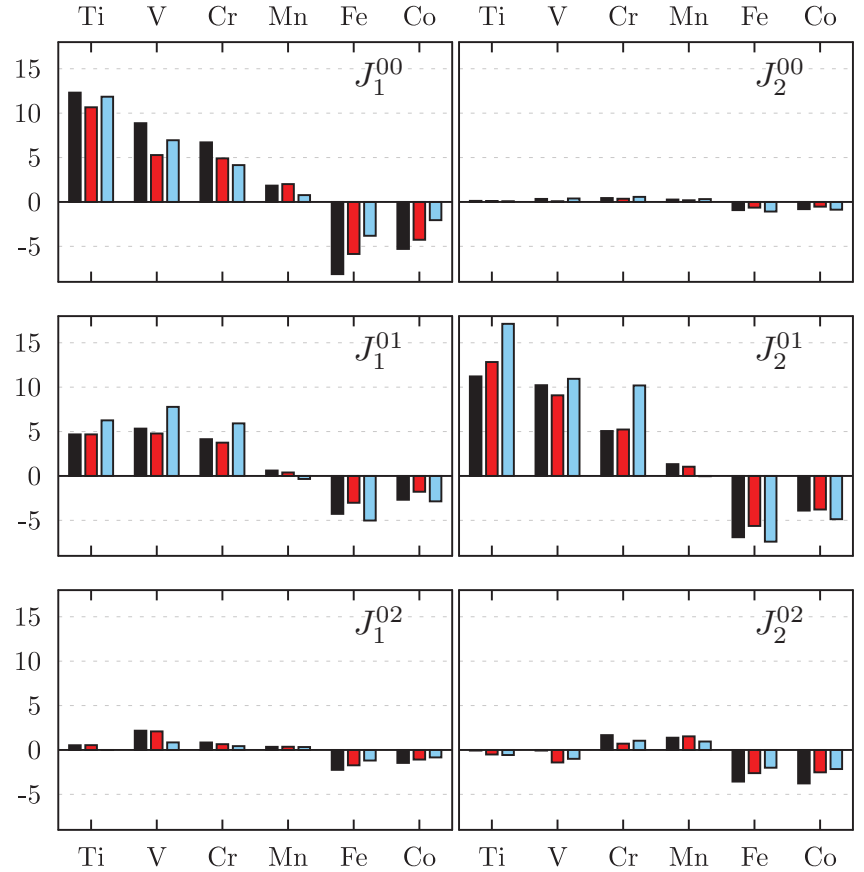

$\mathrm{Bi}_{1.8} \mathrm{TM}_{0.2} \mathrm{Se}_{3} \square \mathrm{Bi}_{1.8} \mathrm{TM}_{0.2} \mathrm{Te}_{3} \square \mathrm{Sb}_{1.8} \mathrm{TM}_{0.2} \mathrm{Te}_{3}$

FIG. 2. (Color online) Exchange constants (in meV) in magnetic $\mathrm{Bi}_{1.80} \mathrm{TM}_{0.20} \mathrm{Se}_{3}, \mathrm{Bi}_{1.80} \mathrm{TM}_{0.20} \mathrm{Te}_{3}$, and $\mathrm{Sb}_{1.80} \mathrm{TM}_{0.20} \mathrm{Te}_{3}(\mathrm{TM}=\mathrm{Ti}, \mathrm{V}$, $\mathrm{Cr}, \mathrm{Mn}, \mathrm{Fe}, \mathrm{Co}$ ). See the schematic view of magnetic interactions in Fig. 1.

being concentrated mostly around the Fermi level with one occupied $d$ orbital, while the $d$ bands in the minority spin channel are all unoccupied. Thus, the net magnetization is $1 \mu_{\mathrm{B}}$ per atom, indicating a valency of $3+$ for each Ti atom. The sharp DOS at the Fermi energy leads to a large ferromagnetic coupling between the nearest magnetic moments within the $\mathrm{Sb}$ plane (Fig. 2).

(ii) With increasing number of $d$ electrons per TM atom, the exchange splitting becomes larger (see Fig. 3). This leads to a strong decrease of the exchange interactions in $\mathrm{Sb}_{2-x} \mathrm{Mn}_{x} \mathrm{Te}_{3}$, in which the majority and minority spin- $d$ electrons are well separated in energy and show minor dispersion. In the case of Fe impurities, due to an occupied minority spin- $d$ state at the Fermi level, the magnitude of the exchange interaction increases but becomes negative because of the large exchange splitting and the isolated impurity-like character of the occupied $d$ orbitals. For Co and Ni impurities, the valency changes from $3+$ to $2+$ and $1+$, respectively. This reduces the magnitude of the exchange interaction, which remains negative. The exchange interaction of $\mathrm{Sb}_{2-x} \mathrm{Ni}_{x} \mathrm{Te}_{3}$, $\mathrm{Bi}_{2-x} \mathrm{Ni}_{x} \mathrm{Te}_{3}$, and $\mathrm{Bi}_{2-x} \mathrm{Ni}_{x} \mathrm{Se}_{3}$ is extremely weak and is not discussed here.

(iii) For almost all cases, the strongest exchange interaction is found between magnetic moments located in different $\mathrm{Sb}$ (Bi) planes but within the same QL $\left(J_{2}^{01}\right.$ in Figs. 1 and 2). The coupling weakens systematically with the number of $d$ electrons. This interaction occurs via a $\mathrm{Te}(\mathrm{Se})$ atom lying between two impurities and is of double exchange type. In addition, the magnitude of $J_{1}^{01}$ is as large as for the in-plane interaction between the nearest magnetic moments, which is an indirect exchange interaction mediated by freecarrier $s p$ states [46]. We thus conclude that two different exchange mechanisms, the double exchange interaction via an anion and the indirect exchange coupling via free carriers, determine the magnetic order in the TM-doped tetradymite chalcogenides.
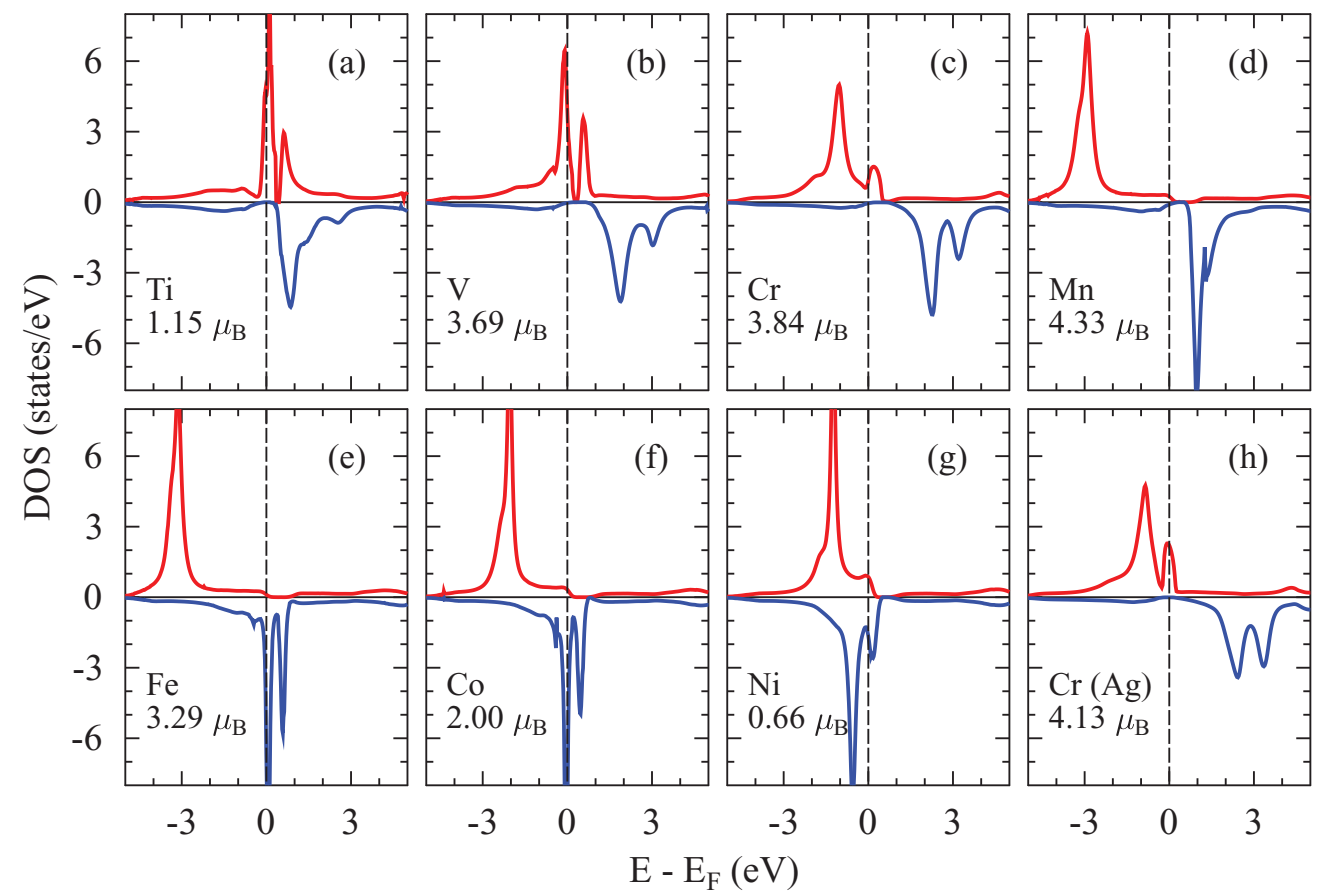

FIG. 3. (Color online) Spin resolved DOS of the TM atoms in $\mathrm{Sb}_{1.8} \mathrm{TM}_{0.2} \mathrm{Te}_{3}$ [(a) $\mathrm{TM}=\mathrm{Ti}$, (b) V, (c) Cr, (d) $\mathrm{Mn}$, (e) Fe, (f) Co, and (g) $\mathrm{Ni}$. Panel (h) shows the spin-resolved DOS of $\mathrm{Cr}$ in $\mathrm{Ag}_{0.25} \mathrm{Sb}_{1.8} \mathrm{Cr}_{0.2} \mathrm{Te}_{3}$. Red (blue) line stands for the majority (minority) spin channel. The vertical dashed lines indicate the Fermi energy. 


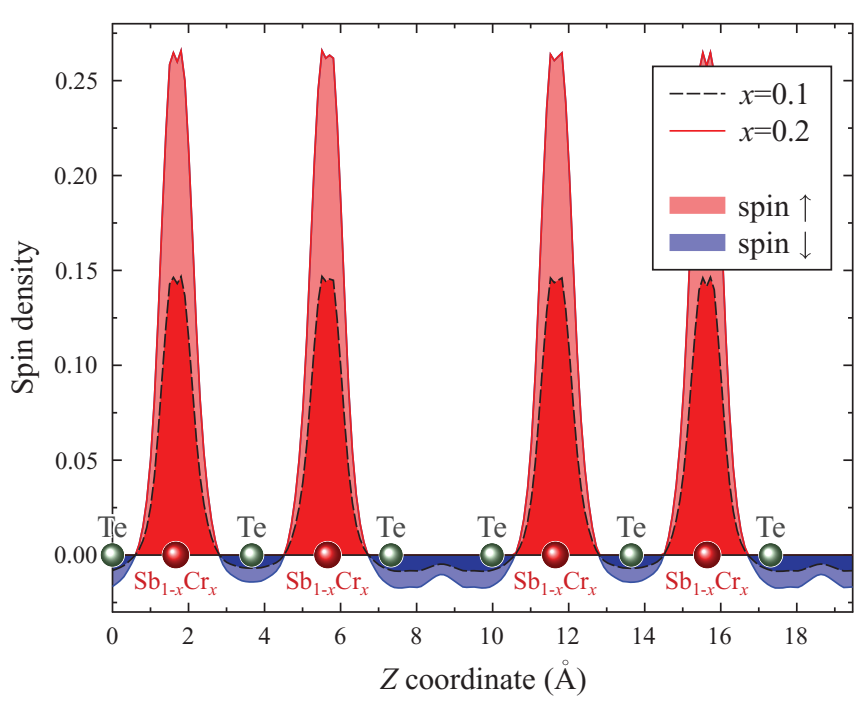

FIG. 4. (Color online) Spin density $m(z)$ (see text) of $\mathrm{Sb}_{2-x} \mathrm{Cr}_{x} \mathrm{Te}_{3}$ for $x=0.1$ and $x=0.2$ in the [0001] direction integrated over all in-plane coordinates $x$ and $y$. The $z$ range covers two QLs.

(iv) The size of cation atoms is crucial for the exchange interaction. The large size of atoms and, thus, the more spatially extended wave functions can lead to a strong hybridization with the electronic states of the neighboring atoms. On the one hand, this can increase the number of free carriers within the cation layer, favoring the indirect exchange of Zener type [46]. On the other hand, the strong binding between a cation (e. g., Bi) and an anion (e. g., Te) reduces the number of valence electrons of the anion and thereby reduces the strength of the double-exchange interaction. Therefore, in the case of $\mathrm{Sb}_{2} \mathrm{Te}_{3}$, the double-exchange interaction via $\mathrm{Te}$ atoms is significantly larger than that in $\mathrm{Bi}_{2} \mathrm{Te}_{3}$ and $\mathrm{Bi}_{2} \mathrm{Se}_{3}$.

(v) Surprisingly the exchange interaction between magnetic moments located in neighboring QLs does not vanish (see $J_{1}^{02}$ and $J_{2}^{02}$ in Figs. 1 and 2). The spin density $m(z) \equiv$ $\left[\rho_{\uparrow}(z)-\rho_{\downarrow}(z)\right] /\left[\rho_{\uparrow}(z)+\rho_{\downarrow}(z)\right]\left[\right.$ where $\rho_{\uparrow}(z)$ and $\rho_{\downarrow}(z)$ stand for the spin-up and spin-down charge densities, respectively, integrated over the lateral coordinates $x$ and $y$ ] "bridges" the van der Waals gap and is responsible for the "inter-QL" magnetic interaction (Fig. 4). The spin density in anion layers is negative and has a magnitude comparable with that of the spin density in the van der Waals gap.

(vi) The amplitude of the exchange interaction is found to decrease with the increase of dopant concentration, which is typical for diluted magnetic semiconductors $[47,48]$.

\section{B. Estimation of critical temperatures}

Considering a wider range of concentrations of the TM atoms, up to $x=1.0$, we estimated the critical temperatures $T_{\mathrm{C}}$ using a Monte Carlo method [49-51]. To treat both ferromagnetic and antiferromagnetic materials, we investigate the spin-spin-correlation function

$$
S=\sum_{i} \sum_{j \in \Omega_{i}}\left|\vec{m}_{i} \cdot \vec{m}_{j}\right|,
$$
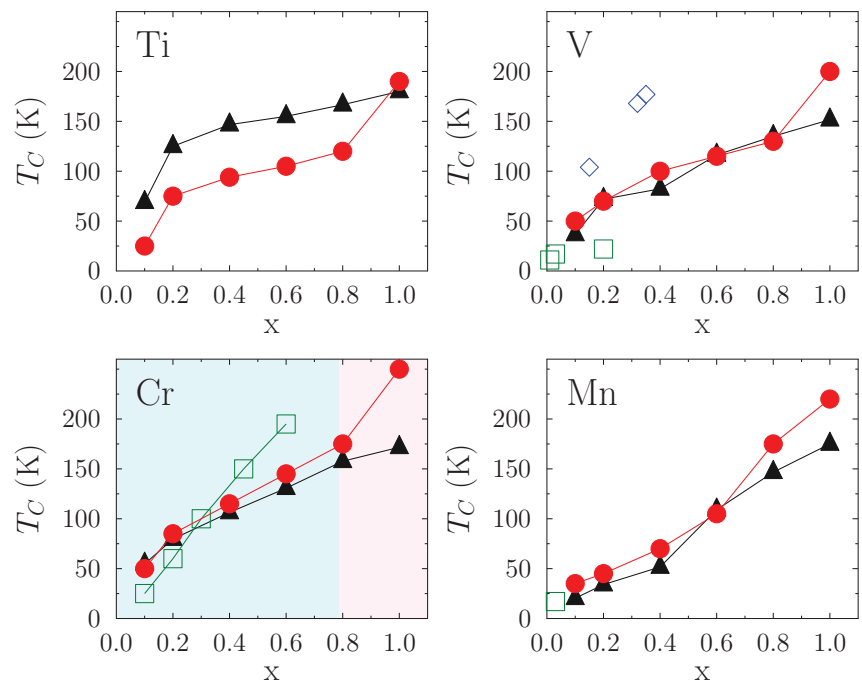

FIG. 5. (Color online) Calculated critical temperature $T_{\mathrm{C}}$ versus concentration $x$ of $\mathrm{Ti}, \mathrm{V}, \mathrm{Cr}$, and $\mathrm{Mn}$ in $\mathrm{Sb}_{2-x} \mathrm{TM}_{x} \mathrm{Te}_{3}$. The critical temperature $T_{\mathrm{C}}$ is determined from Monte Carlo simulations with randomly distributed impurities (black-filled triangles) and with cluster percolation (red-filled circles). $T_{\mathrm{C}}$ is compared to experimental data (green and blue markers) [19,20]. In the case of $\mathrm{Cr}$, there is a transition from a ferromagnetic state for $x<0.8$ (light-blue background) to an antiferromagnetic state at higher concentrations (light-red background).

where $\vec{m}_{i}$ and $\Omega_{i}$ are the magnetic moment and the interaction sphere around site $i$, respectively. We also account for percolation effects, using pair potentials, and compared estimated critical temperatures with the available experimental data. The results for ferromagnetic $\mathrm{Sb}_{2-x} \mathrm{TM}_{x} \mathrm{Te}_{3}(\mathrm{TM}=\mathrm{Ti}$, V, Cr, Mn; Fig. 5) show a systematic increase of the $T_{\mathrm{C}}$ with the concentration of dopant. Percolation effects do not affect strongly the behavior of $T_{\mathrm{C}}$ at low concentrations; except in the case of $\mathrm{Ti}$, for which percolation lowers $T_{\mathrm{C}}$. Calculations for $\mathrm{Cr}$ reproduce the experimentally measured trends for concentrations up to $x=0.6$ [20]. For higher concentrations, we found a transition to antiferromagnetic order (area with a light-red background in Fig. 5), which is understood as the result of an increasing antiferromagnetic interaction between magnetic moments from nearby QLs both containing on average an impurity. This explains why experimental data is unavailable for concentrations larger than $x=0.6$.

Concerning the $\mathrm{Sb}_{2-x} \mathrm{~V}_{x} \mathrm{Te}_{3}$ case, we reproduce the trend found in experiments for a broad concentration range [19]. However, the theoretical absolute values are smaller than the experimental ones by about a factor of $1 / 2$. We suppose that either structural imperfections due to sample preparations or limitations in the $a b$ initio description could cause this mismatch. For the reported cases of Mn doping at lowconcentration regimes $(x \leqslant 0.1)$, our calculations are, again, in qualitative agreement with experiment [14,18,23,52-54].

\section{Tuning the exchange interaction}

The systematic study of the exchange interaction in magnetically doped tetradymite chalcogenides presented in this work can open new possibilities for first-principles material design. 
TABLE I. Exchange coupling constants $J_{i}^{0 j}$ (in $\mathrm{meV}$ ) for $\mathrm{Bi}_{1.9-y} \mathrm{Cr}_{0.1} \mathrm{Sb}_{y} \mathrm{Te}_{3}, y=0,0.2$ and 0.4 . Here index $i$ labels magnetic neighbors, belonging to the same $(j=0)$, to the nearest $(j=1)$ or the next-nearest $(j=2)$ atomic plane (see Fig. 1).

\begin{tabular}{|c|c|c|c|}
\hline & $y=0$ & $y=0.2$ & $y=0.4$ \\
\hline & \multicolumn{3}{|c|}{ Intralayer coupling } \\
\hline$J_{1}^{00}$ & 4.91 & 4.79 & 4.66 \\
\hline$J_{2}^{00}$ & 0.36 & 0.37 & 0.37 \\
\hline \multirow[t]{2}{*}{$J_{3}^{00}$} & 0.81 & 0.79 & 0.78 \\
\hline & \multicolumn{3}{|c|}{ Interlayer coupling inside QL } \\
\hline$J_{1}^{01}$ & 3.75 & 3.88 & 4.00 \\
\hline$J_{2}^{01}$ & 5.23 & 5.59 & 5.94 \\
\hline \multirow[t]{2}{*}{$J_{3}^{01}$} & 0.32 & 0.33 & 0.34 \\
\hline & \multicolumn{3}{|c|}{ Inter-QL coupling (nearest atomic layers) } \\
\hline$J_{1}^{02}$ & 0.65 & 0.62 & 0.58 \\
\hline$J_{2}^{02}$ & 0.71 & 0.72 & 0.72 \\
\hline$J_{3}^{02}$ & -7.02 & -6.27 & -5.60 \\
\hline
\end{tabular}

We infer for instance that one way to control the magnetic interaction and the Curie temperature of these materials is to replace particular atoms or sheets of atoms, in order to tune the strength of the electronic hybridization because it is responsible for the exchange-interaction mechanisms in this class of materials. Here, it has been shown that the overlap between electronic wave functions of anions and cations is crucial.

In an experimental realization, one could replace the anion layer between two cation sheets by the layer of atoms of the same group in the periodic table. As an example, let us consider the central anion sheet of each $\mathrm{Sb}_{1.80} \mathrm{Cr}_{0.20} \mathrm{Te}_{3} \mathrm{QL}$ to be formed by various chalcogen atoms. We observe an increase of the associated Curie temperature with ionic size and spatial extension of the electronic wave function of the chalcogen ion: $\mathrm{S}, \mathrm{Se}, \mathrm{Te}$, and Po yield $T_{\mathrm{C}}=69,71,76$, and $79 \mathrm{~K}$, respectively. A similar effect can be achieved with specific co-doping of the corresponding anion layer.

The in-plane exchange interaction can instead be tuned by co-doping of the cation layers in accordance with the exchange-constants behavior presented in Fig. 2. To illustrate this aspect, we calculated the exchange interaction in $\mathrm{Bi}_{1.9-y} \mathrm{Cr}_{0.1} \mathrm{Sb}_{y} \mathrm{Te}_{3}(y=0.0,0.2$, and 0.4$)$, where the cation layers are co-doped by different amounts of $\mathrm{Sb}$ atoms. As one can see from Table I, the in-plane interaction $\left(J_{1}^{00}\right)$ decreases with the increase of Sb concentration due to the smaller cation size, while the double-exchange interaction $\left(J_{2}^{01}\right)$ increases owing to a higher electron mobility. Thus we find a competition of the two magnetic contributions that results in an increase of Curie temperature from $35 \mathrm{~K}$ at $y=0$ to 39 and $42 \mathrm{~K}$ at $y=0.2$ and 0.4 , respectively.

Another way to tune the magnetic interaction is to insert impurities into the van der Waals gaps [55]. This can change the separation between QLs and, by a proper choice of impurities, can supply free carriers, which are important for the indirect exchange of Zener type. Such a tuning is demonstrated for Agintercalated $\mathrm{Sb}_{1.8} \mathrm{Cr}_{0.2} \mathrm{Te}_{3}$, where the in-plane and out-of-plane exchange interactions are strongly modified by the presence of Ag impurities in the van der Waals gaps (Table II). Ag in the
TABLE II. The same as Table I, but for $\mathrm{Ag}_{y} \mathrm{Sb}_{1.8} \mathrm{Cr}_{0.2} \mathrm{Te}_{3}, y=0$ and 0.25 .

\begin{tabular}{lrr}
\hline \hline & $y=0$ & $y=0.25$ \\
\hline & \multicolumn{2}{c}{ Intralayer coupling } \\
\cline { 2 - 3 }$J_{1}^{00}$ & 4.15 & 0.25 \\
$J_{2}^{00}$ & 0.57 & 0.21 \\
$J_{3}^{00}$ & 0.92 & 0.63 \\
$J_{4}^{00}$ & 0.12 & 0.07 \\
& Interlayer coupling inside QL \\
\cline { 2 - 3 }$J_{1}^{01}$ & 5.92 & 3.61 \\
$J_{2}^{01}$ & 10.19 & 16.02 \\
$J_{3}^{01}$ & 0.65 & 0.47 \\
$J_{4}^{01}$ & 1.58 & 1.39 \\
& Inter-QL coupling (nearest \\
& \multicolumn{2}{c}{ atomic layers) } \\
$J_{1}^{02}$ & 0.43 & 0.21 \\
$J_{2}^{02}$ & 1.04 & 0.88 \\
$J_{3}^{02}$ & -0.09 & 0.05 \\
$J_{4}^{02}$ & -0.05 & 0.20 \\
\hline \hline
\end{tabular}

van der Waals gap acts as a p dopant and shifts the Fermi level decreasing the number of holes [see Figs. 3(c) and 3(h)]. This strongly reduces the exchange interaction of the Zener type. At the same time, the increase of free carries due to the $p$ doping enhances the strength of the double exchange interaction. Indeed, we observe that upon Ag intercalation the in-plane exchange $\left(J_{1}^{00}\right)$ decreases by an order of magnitude, while the double exchange $\left(J_{2}^{01}\right)$ gets stronger by a factor of 1.5 . As a result, the Curie temperature increases from 89 to $95 \mathrm{~K}$.

\section{CONCLUSIONS}

We studied the exchange interaction in the $\mathrm{Bi}_{2} \mathrm{Se}_{3}, \mathrm{Bi}_{2} \mathrm{Te}_{3}$, and $\mathrm{Sb}_{2} \mathrm{Te}_{3}$ tetradymite chalcogenides doped with transition metal atoms. Our first-principles calculations have shown that the magnetic interaction is long-range and is mainly mediated out of plane by the double exchange mechanism via an anion and in-plane by the indirect exchange coupling via free carriers. The calculated Curie temperatures as a function of TM dopant concentration were found to be in qualitative agreement with available experimental data. Finally, we presented several ways to tune the magnetic interaction in these systems: (i) replacing the anion layer between two cation sheets by atoms of the same group, (ii) co-doping of the cation sheet, and (iii) inserting impurities in the van der Waals gap. These results provide deep insight into the magnetic interactions in the magnetic binary tetradymite chalcogenides, and open ways to design new materials for promising applications.

\section{ACKNOWLEDGMENTS}

We acknowledge support by the Tomsk State University Competitiveness Improvement Program and the Deutsche Forschungsgemeinschaft (Priority Program SPP 1666 "Topological Insulators"). The calculations were performed at the Rechenzentrum Garching of the Max Planck Society (Germany) and at the SKIF-Cyberia supercomputer of Tomsk State University. 
[1] G. Nolas, J. Sharp, and J. Goldsmid, Thermoelectrics: Basic Principles and NewMaterials Developments, Springer Series in Materials Science (Springer-Verlag, Berlin, Heidelberg, 2001).

[2] M. Z. Hasan and C. L. Kane, Rev. Mod. Phys. 82, 3045 (2010).

[3] X.-L. Qi and S.-C. Zhang, Rev. Mod. Phys. 83, 1057 (2011).

[4] S. V. Eremeev, G. Landolt, T. V. Menshchikova, B. Slomski, Y. M. Koroteev, Z. S. Aliev, M. B. Babanly, J. Henk, A. Ernst, L. Patthey et al., Nat. Commun 3, 635 (2012).

[5] S. Muff, F. von Rohr, G. Landolt, B. Slomski, A. Schilling, R. J. Cava, J. Osterwalder, and J. H. Dil, Phys. Rev. B 88, 035407 (2013).

[6] T. Okuda, T. Maegawa, M. Ye, K. Shirai, T. Warashina, K. Miyamoto, K. Kuroda, M. Arita, Z. S. Aliev, I. R. Amiraslanov et al., Phys. Rev. Lett. 111, 206803 (2013).

[7] Q. Zhang, Z. Zhang, Z. Zhu et al., ACS Nano 6, 2345 (2012).

[8] T. V. Menshchikova, M. M. Otrokov, S. S. Tsirkin, D. A. Samorokov, V. V. Bebneva, A. Ernst, V. M. Kuznetsov, and E. V. Chulkov, Nano Lett. 13, 6064 (2013).

[9] J. Zhang, C.-Z. Chang, Z. Zhang et al., Nat. Commun. 2 (2011).

[10] T. Arakane, T. Sato, S. Souma et al., Nat. Commun. 3 (2012).

[11] C.-Z. Chang, J. Zhang, M. Liu, Z. Zhang, X. Feng, K. Li, L.-L. Wang, X. Chen, X. Dai, Z. Fang et al., Adv. Mater. 25, 1065 (2013).

[12] C.-Z. Chang, J. Zhang, X. Feng, J. Shen, Z. Zhang, M. Guo, K. Li, Y. Ou, P. Wei, L.-L. Wang et al., Science 340, 167 (2013).

[13] J. S. Dyck, P. Hájek, P. Lošt'ák, and C. Uher, Phys. Rev. B 65, 115212 (2002).

[14] J. S. Dyck, P. Svanda, P. Lošt'ák, J. Horak, W. Chen, and C. Uher, J. Appl. Phys. 94, 7631 (2003).

[15] J. S. Dyck, C. Drašar, P. Lošt'ák, and C. Uher, Phys. Rev. B 71, 115214 (2005).

[16] V. A. Kul'bachinskii, A. Yu. Kaminskii, K. Kindo, Y. Narumi, K. Suga, P. Lostak, and P. Svanda, JETP Lett. 73, 352 (2001).

[17] V. Kulbachinskii, P. Tarasov, and E. Brück, J. Exp. Theor. Phys. 101, 528 (2005).

[18] J. Choi, S. Choi, J. Choi, Y. Park, H.-M. Park, H.-W. Lee, B.-C. Woo, and S. Cho, Phys. Status Solidi B 241, 1541 (2004).

[19] Z. Zhou, Y.-J. Chien, and C. Uher, Appl. Phys. Lett. 87, 112503 (2005).

[20] Z. Zhou, Y.-J. Chien, and C. Uher, Phys. Rev. B 74, 224418 (2006).

[21] Z. Zhou, M. Zabeik, P. Lostak, and C. Uher, J. Appl. Phys. 99, 043901 (2006).

[22] J. W. G. Bos, M. Lee, E. Morosan, H. W. Zandbergen, W. L. Lee, N. P. Ong, and R. J. Cava, Phys. Rev. B 74, 184429 (2006).

[23] Y. S. Hor, P. Roushan, H. Beidenkopf, J. Seo, D. Qu, J. G. Checkelsky, L. A. Wray, D. Hsieh, Y. Xia, S.-Y. Xu et al., Phys. Rev. B 81, 195203 (2010).

[24] D. Hsieh, Y. Xia, D. Qian, L. Wray, F. Meier, J. H. Dil, J. Osterwalder, L. Patthey, A. V. Fedorov, H. Lin et al., Phys. Rev. Lett. 103, 146401 (2009).
[25] J. Honolka, A. A. Khajetoorians, V. Sessi, T. O. Wehling, S. Stepanow, J.-L. Mi, B. B. Iversen, T. Schlenk, J. Wiebe, N. B. Brookes et al., Phys. Rev. Lett. 108, 256811 (2012).

[26] L. R. Shelford, T. Hesjedal, L. Collins-McIntyre, S. S. Dhesi, F. Maccherozzi, and G. van der Laan, Phys. Rev. B 86, 081304 (2012).

[27] D. West, Y. Y. Sun, S. B. Zhang, T. Zhang, X. Ma, P. Cheng, Y. Y. Zhang, X. Chen, J. F. Jia, and Q. K. Xue, Phys. Rev. B 85, 081305 (2012).

[28] M. R. Scholz, J. Sánchez-Barriga, D. Marchenko, A. Varykhalov, A. Volykhov, L. V. Yashina, and O. Rader, Phys. Rev. Lett. 108, 256810 (2012).

[29] X. F. Kou, W. J. Jiang, M. R. Lang, F. X. Xiu, L. He, Y. Wang, Y. Wang, X. X. Yu, A. V. Fedorov, P. Zhang et al., J. Appl. Phys. 112, 063912 (2012).

[30] M. Ye, S. V. Eremeev, K. Kuroda, E. E. Krasovskii, E. V. Chulkov, Y. Takeda, Y. Saitoh, K. Okamoto, S. Y. Zhu, K. Miyamoto et al., Phys. Rev. B 85, 205317 (2012).

[31] P. Larson and W. R. L. Lambrecht, Phys. Rev. B 78, 195207 (2008).

[32] R. Yu, W. Zhang, H.-J. Zhang, S.-C. Zhang, X. Dai, and Z. Fang, Science 329, 61 (2010).

[33] J.-M. Zhang, W. Zhu, Y. Zhang, D. Xiao, and Y. Yao, Phys. Rev. Lett. 109, 266405 (2012).

[34] J. Henk, A. Ernst, S. V. Eremeev, E. V. Chulkov, I. V. Maznichenko, and I. Mertig, Phys. Rev. Lett. 108, 206801 (2012).

[35] J. Henk, M. Flieger, I. V. Maznichenko, I. Mertig, A. Ernst, S. V. Eremeev, and E. V. Chulkov, Phys. Rev. Lett. 109, 076801 (2012).

[36] J. P. Perdew and Y. Wang, Phys. Rev. B 45, 13244 (1992).

[37] U. von Barth and L. Hedin, J. Phys. C 5, 1629 (1972).

[38] J. Korringa, Physica 13, 392 (1947).

[39] W. Kohn and N. Rostoker, Phys. Rev. 94, 1111 (1954).

[40] M. Lüders, A. Ernst, W. M. Temmerman, Z. Szotek, and P. J. Durham, J. Phys. Condens. Matter 13, 8587 (2001).

[41] S. M. Souza, C. M. Poffo, D. M. Trichês, J. C. de Lima, T. A. Grandi, A. Polian, and M. Gauthier, Phys. B (Amsterdam, Neth.) 407, 3781 (2012).

[42] V. V. Atuchin, T. A. Gavrilova, K. A. Kokh, N. V. Kuratieva, N. V. Pervukhina, and N. V. Surovtsev, Solid State Commun. 152, 1119 (2012).

[43] C. Pérez Vicente, J. L. Tirado, K. Adouby, J. C. Jumas, A. Abba Touré, and G. Kra, Inorg. Chem. 38, 2131 (1999).

[44] B. L. Gyorffy, Phys. Rev. B 5, 2382 (1972).

[45] A. I. Liechtenstein, M. I. Katsnelson, V. P. Antropov, and V. A. Gubanov, J. Magn. Magn. Mater. 67, 65 (1987).

[46] C. Zener, Phys. Rev. 81, 440 (1951).

[47] J. Kudrnovský, V. Drchal, I. Turek, L. Bergqvist, O. Eriksson, G. Bouzerar, L. Sandratskii, and P. Bruno, J. Phys.: Condens. Matter 16, S5571 (2004).

[48] K. Sato, L. Bergqvist, J. Kudrnovský, P. H. Dederichs, O. Eriksson, I. Turek, B. Sanyal, G. Bouzerar, H. KatayamaYoshida, V. A. Dinh et al., Rev. Mod. Phys. 82, 1633 (2010).

[49] N. Metropolis, A. W. Rosenbluth, M. N. Rosenbluth, and E. Teller, J. Chem. Phys. 21, 1087 (1953).

[50] K. Binder, Rep. Prog. Phys 60, 487 (1997). 
[51] G. Fischer, M. Dane, A. Ernst, P. Bruno, M. Luders, Z. Szotek, W. Temmerman, and W. Hergert, Phys. Rev. B 80, 014408 (2009).

[52] J. Choi, H.-W. Lee, B.-S. Kim, S. Choi, J. Choi, J. H. Song, and S. Cho, J. Appl. Phys. 97, 10D324 (2005).

[53] J. Choi, H.-W. Lee, B.-S. Kim, H. Park, S. Choi, S. Hong, and S. Cho, J. Magn. Magn. Mater. 304, e164 (2006).
[54] Y. H. Choi, N. H. Jo, K. J. Lee, H. W. Lee, Y. H. Jo, J. Kajino, T. Takabatake, K.-T. Ko, J.-H. Park, and M. H. Jung, Appl. Phys. Lett. 101, 152103 (2012).

[55] M. M. Otrokov, S. D. Borisova, V. Chis, M. G. Vergniory, S. V. Eremeev, V. M. Kuznetsov, and E. V. Chulkov, JETP Lett. 96, 714 (2013). 